\title{
Metodología 4321 de trabajo en grupo para fortalecer el aprendizaje de la programación usando aprendizaje colaborativo y aprendizaje cooperativo
}

\author{
Omar Iván Trejos-Buriticá, \\ Facultad de Ingenierías, Universidad Tecnológica de Pereira, Pereira, Colombia. omartrejos@utp.edu.co
}

\begin{abstract}
Resumen-- El propósito de la investigación que inspira este artículo consiste en la búsqueda de caminos que permitan aprovechar las fortalezas que puede proporcionarle, a un estudiante promedio, el trabajo en grupo desde una perspectiva tanto colaborativa como cooperativa, dependiendo de las necesidades. Este estudio se ha realizado en el $1^{\circ}$ semestre de Ingeniería de Sistemas a lo largo de tres años y para ello se ha acudido a una metodología tanto cuantitativa como cualitativa a partir de los resultados numéricos de las evaluaciones como de la interacción, observación y diálogo con los estudiantes. Se han obtenido unos resultados que permiten aportar a la discusión de trabajo en grupo y que proporcionan elementos de juicio para aprovechar lo que se haga en el aula a la luz de esta técnica. Se concluye que es posible lograr que se mejore el aprendizaje individual de la programación de computadores si el trabajo en grupo se estructura bajo una metodología claramente definida, si se aplica un enfoque que se ajuste a las necesidades de lo que se quiere hacer y si se logra que los estudiantes sean partícipes activos de este tipo de procesos.
\end{abstract}

Palabras clave-- Aprendizaje colaborativo; aprendizaje cooperativo; enseñanza de la programación; metodología de trabajo; programación de computadores.

Recibido para revisar Junio 5 de 2017, aceptado Agosto 9 de 2017, versión final Agosto 20 de 2017

\section{Methodology teamwork's 4321 to improve learning in computer programming using collaborative learning and cooperative learning}

\begin{abstract}
The purpose of the research this article is based is the finding of some ways that let us to improve the programming computer learning using the strengths of a working group for an average student using collaborative and cooperative learning. This research was developed in the $1^{\text {st }}$ semester of Systems Engineer program during three years and we use a quantitative and qualitative methodology with numerical results in the assessments and interaction and communication with the students. We obtained results for the discussion about the working groups that bring some criteria for the academic work in the classroom. We conclude that is possible to improve the personal learning of the computer programming if the work in groups is bases in a defined methodology, if we use also a defined approach about we want in the classroom and if the students participate actively in this process.
\end{abstract}

Keywords-- Collaborative Learning; cooperative learning; programming teaching; working methodology; computer programming.

\section{Introducción}

Una de las grandes dificultades que se presentan en el desarrollo del proceso formativo de los estudiantes de programas de Ingeniería y, especialmente, de Ingeniería de
Sistemas consiste en poder articular habilidades y cualidades para que se trabaje en grupo y para que éstos se conviertan en equipos que complementen sus habilidades en el logro de diferentes objetivos. Normalmente el trabajo en grupo es una de las estrategias más cotidianas en los salones de clase de las Universidades pero ¿cómo se desarrollan las actividades que se realizan con grupos de estudiantes? ¿cuál podría ser un camino para lograr que, por la vía del trabajo en grupo, se puedan complementar las habilidades y, al interior de un grupo, unos integrantes puedan aprender de otros?

Estas son apenas algunas de las inquietudes que han inspirado esta investigación que, junto con otras, propende por conocer algunas técnicas para que el trabajo en grupo sea eficiente y permite el crecimiento cognitivo y profesional de cada uno de los integrantes. A este respecto debe advertirse que se han desarrollado diferentes estudios orientados a capitalizar técnicas de trabajo en grupo con el ánimo de poder lograr los mejores resultados tanto para el grupo como para cada uno de los integrantes de manera individual.

¿Cuántas veces el resultado de un grupo muy satisfactorio pero el resultado individual no lo es tanto?¿cuántas veces se ha visto en el salón de clase que un grupo entrega todo lo previsto dentro de una actividad específica pero, cuando ésta se confronta con los resultados individuales, se observa que ese mismo trabajo en grupo (aparentemente exitoso) no ha tenido el efecto esperado en cada uno de los integrantes? Lo que se pretende en este artículo es presentar los resultados de una metodología que busca articular a los estudiantes en una conformación adecuada de los grupos basándose en un modelo especifico de caracterización de habilidades y enfoques y, de la misma manera, posibilitar el aprendizaje de unos y otros de manera que, al final, cada uno haya logrado sus objetivos individuales dentro de un proceso de formación en ingeniería.

Se ha acudido a una clasificación de estudiantes basada en el modelo $4 \mathrm{Q}$ de preferencias de pensamiento formulada por el Dr. William Herrmann (Herrmann, 2000) para que los grupos no se organicen de manera aleatoria sino que se haga desde una perspectiva científica de forma que sus habilidades se puedan complementar en función de los objetivos de aprendizaje y que éstos se alcancen tanto en lo grupal como en lo individual. A lo

Como citar este artículo: Trejos-Buriticá, O.I., Metodología 4321 de trabajo en grupo para fortalecer el aprendizaje de la programación usando aprendizaje colaborativo y aprendizaje cooperativo. Educación en Ingeniería, 13(25), pp. 28-36, Febrero, 2018. 
largo del semestre se planearon cuatro evaluaciones: tres parciales y una evaluación final y se realizaron en formato de grupos según la clasificación establecida. También se realizaron talleres cuya valoración formaba parte integral de cada uno de los parciales. En la sección destinada a tal fin se presentarán los resultados pertinentes.

Los resultados indicaron que definitivamente cuando se cuenta con un modelo específico para organizar los grupos de trabajo de los estudiantes, es posible esperar un impacto más favorable al proceso individual de cada uno, lo cual invita a pensar que el trabajo en grupo va mucho más allá de la simple agrupación de estudiantes, sino que corresponde al docente el perfilamiento de ellos para que, sobre la base de sus capacidades individuales y sus ritmos personales de aprendizaje, se pueda conformar grupos que sean complementarios al interior de cada uno. El docente no solo debe ser un guía sino un organizador y un coequipero. Como organizador tiene la tarea de conocer modelos que permitan perfilar a los estudiantes y como coequipero debe estar en la capacidad de acompañarlos, de analizarlos, de complementar sus necesidades de aprendizaje y, en lo posible, de resolverlas de manera satisfactoria.

Lo innovador de esta investigación podría estar en el hecho de que pocas veces se acude a modelos científicos para la conformación de grupos y para el análisis de su desempeño y que no siempre se toman el tiempo necesario, los docentes ingenieros, para comparar resultados e intentar encontrar caminos más eficaces para el proceso de aprendizaje que conducen, además de probar las conclusiones en el aula de clases.

Este artículo es un producto del proyecto de investigación 6-16-13 titulado "Desarrollo de un modelo metodológico para el aprendizaje de la programación en Ingeniería de Sistemas basado en Aprendizaje Significativo, Aprendizaje por Descubrimiento y el Modelo 4Q de preferencias de pensamiento" avalado por el Consejo de Facultad de Ingenierías y aprobado por la Vicerrectoría de Investigaciones, Extensión e Innovación de la Universidad Tecnológica de Pereira.

La temática que se propone como su razón investigativa de ser plantea especial relevancia por el posible impacto que puede generar en ingenieros docentes $y$, en general, en aquellos docentes de programas de formación tecnológica. La investigación se desarrolló con los estudiantes del curso Programación II de Ingeniería de Sistemas y Computación de la Facultad de Ingenierías de la Universidad Tecnológica de Pereira durante los semestres I 2014, II 2014, I 2015, II 2015, I 2016 y II 2016. El presente estudio se ha realizado bajo los parámetros y metodologías asociadas con la investigación cuantitativa y cualitativa en Educación y se ha acudido a las teorías de aprendizaje significativo y aprendizaje por descubrimiento sobre la base de los enfoques active learning, collaborative learning, cooperative learning y el modelo $4 \mathrm{Q}$ de preferencias de pensamiento.

¿Es posible potencializar las capacidades y los ritmos de aprendizaje individuales de los estudiantes de un curso de programación de computadores a partir de una metodología de trabajo en grupos que se han conformado con criterios científicos y que se han organizado bajo metodologías inspiradas en teorías de aprendizaje? La respuesta es el contenido de este artículo que se espera aporte elementos de juicio para las discusiones que, al respecto, tengan lugar en las salas de profesores de programas de Ingeniería.

El objetivo general de este artículo, según lo expuesto, es aportar elementos de juicio en el sentido de la hipótesis e intentar encontrar caminos que capitalicen el trabajo en grupo a la luz de un curso de programación. Para el desarrollo tanto de la investigación como del artículo se ha acudido a una bibliografía especializada y a los aportes que proporciona la experiencia del autor. El artículo está organizado según la estructura IMRYD que inicia con una introducción que complementada por un marco teórico.

Luego se expone la metodología que se utilizó en la investigación y se presentan los resultados derivados de la misma para realizar una discusión a partir de ellos. Finalmente se plantean unas conclusiones que se espera enriquezcan las discusiones al respecto de la formación en programación en Ingeniería de Sistemas y otras ingenierías y el trabajo en el aula bajo la metodología de trabajos en grupo.

\section{Marco Teórico}

El aprendizaje significativo se refiere a una teoría de aprendizaje [1] según la cual el conocimiento se adquiere a partir de las relaciones que se puedan establecer con el conocimiento previo. A la luz de esta teoría, los aprendices se integran activamente con el nuevo conocimiento teniendo en cuenta las bases que les proporciona el conocimiento previo que ya traen. Los mapas conceptuales han permitido, graficamente, convertirse en una tecnica que permite a los estudiantes conectar el conocimiento preexistente en determinada asignatura [2]. Posiblemente sea el Internet el factor principal que actualmente incide en el aprendizaje significativo. Las tecnologías Web 2.0, tales como Wikipedia, los blogs y Youtube, han logrado que el aprendizaje sea mucho mas sencillo y han posibilitado un acceso mas fácil a los estudiantes [3].

Los estudiantes han demostrado mayor interés en la medida en que tienen acceso libre y fácil a estas herramientas, y por lo tanto pueden aprender con mayor facilitad cuando el material tiene significado frente a sus necesidades de conocimiento. El desarrollo de la motivación es uno de los objetivos del aprendizaje significativo dado que los estudiantes que estén más interesados aprenden de manera más efectiva [4]. A partir de la teoría cognitiva del aprendizaje, basada en la teoría del procesamiento humano de la información, los tres principales procesos de aprendizaje son: cómo se desarrolla el conocimiento, cómo se integra el nuevo conocimiento dentro de un sistema de conocimiento ya existente y cómo el conocimiento se incorpora automáticamente.

Ausubel se enfocó en el aprendizaje significativo, como una experiencia clara y precisa que se articula conscientemente con signos, símbolos, conceptos y proposiciones que tienen significado y que aportan elementos individuales para fortalecer la estructura cognitiva a partir del enlace posible entre conocimiento previo, el nuevo conocimiento y la actitud del estudiante. Cuando se habla de la misión de un proceso de aprendizaje, es posible que muchos estén de acuerdo en que éste debe permitir que el conocimiento vaya más allá de las creencias, las costumbres y los valores [5].

El propósito de la formación profesional consiste en preparar a los estudiantes para los retos y necesidades que se 
encuentran en la sociedad. Por lo tanto se necesita que el conocimiento no sea un conjunto de datos e información que esté aislada del contexto en el cual se necesita sino que los estudiantes puedan establecer nexos entre lo que aprenden y lo que viven de tal forma que, al encontrar significado, puedan hacer efectivos los conocimientos adquiridos desde sus respectivos procesos de formación profesional [6].

Cuando se aprende, se asocian significados que tienen que ver con las cosas que están alrededor nuestro, y esos significados pueden ser diferentes dependiendo del entorno cultural [7]. Uno de los temas educativos recurrentes es el debate acerca de lo que sabemos acerca del aprendizaje significativo y la capacidad individual de aplicarlo en diferentes contextos. La pregunta que surge entonces es ¿qué grado de significatividad en el aprendizaje depende de la relación que los estudiantes puedan establecer entre el nuevo conocimiento y el conocimiento previo? El conocimiento es considerado significativo cuando se puede generalizar, cuando puede ser aplicar y cuando es durable en el tiempo. Un conocimiento durable es aquel que perdura por un largo tiempo en la memoria $\mathrm{y}$ al cual podemos acceder en cualquier momento [8]. Que el conocimiento se pueda generalizar significa que se puede asociar a diferentes contextos, situaciones y tareas y que sea aplicable o funcional significa que lo aprendido nos permita actuar de manera diferente.

El aprendizaje por descubrimiento es una teoría de aprendizaje con un enfoque constructivista que está soportada por los trabajos científicos de sicólogos como Jean Piaget y Jerome Seymour Bruner. Esta filosofía originó en su momento el movimiento de aprendizaje por descubrimiento, el cual se fundamenta en algo muy simple, “aprender haciendo”.

La estructura del aprendizaje por descubrimiento puede cubrir diferentes técnicas instruccionales. Una tarea de aprendizaje puede ir desde el hallazgo de un patrón implícito hasta las explicaciones más detalladas de un manual de simulaciones [9]. El aprendizaje por descubrimiento puede ocurrir cuando el estudiante aún no tiene una respuesta exacta pero cuenta con los materiales para encontrarla si los utiliza apropiadamente y de manera autónoma. Éste emerge en las situaciones donde debe resolverse un problema en la cual el aprendiz esquematiza la solución a partir de su propia experiencia y sus conocimientos previos y se convierte en un método de instrucción que permite que los estudiantes interactúen con su entorno a partir de la exploración y manipulación de objetos, luchando inquietudes y controversias o diseñando experimentos [10].

En términos de esta teoría, el aprendiz requiere descubrir en nuevos contextos a través de procesos de investigación o realizando procedimientos que requieren de una pequeña ayuda. Algunos investigadores aseguran que el aprendizaje por descubrimiento sin acompañamiento no necesariamente permite que los estudiantes concluyan las reglas necesarias para resolver problemas, para encontrar estrategias de conservación o para inferir conceptos de programación.

Por su parte, como Active Learning, se conoce un método de enseñanza que invita a que los estudiantes se involucren directa y conscientemente en su propio proceso de aprendizaje [11]. El término "Active Learning” fue introducido recientemente como una forma de invitar a que los estudiantes participen en el proceso de aprendizaje en vez de ser asistentes pasivos del mismo. Es de anotar que los diferentes niveles de Aprendizaje Activo dependerán del compromiso que cada alumno adquiera consigo mismo, con la fijación de sus metas, con los esfuerzos que haga para alcanzarlas y con la efectividad con que lo logre.

Es un modelo de instrucción que enfoca la responsabilidad del aprendizaje en los mismos aprendices. Se han discutido diferentes metodologías para promover el aprendizaje activo teniendo en cuenta que, para aprender, los estudiantes deben hacer mucho más que simplemente escuchar. Deben leer, escribir, discutir o dejarse involucrar por la búsqueda en la solución de problemas. Se relaciona con los tres dominios de aprendizaje referidos como conocimiento, habilidades y actitudes y con la taxonomía del comportamiento del aprendizaje que puede ser enseñado como los objetivos del proceso de aprendizaje [12]. De manera particular, los estudiantes deben ser "enganchados" en tareas de pensamiento de alto nivel tales como análisis, síntesis y evaluaciones. El aprendizaje activo involucra a los estudiantes en dos aspectos: haciendo cosas y pensando en las cosas que están haciendo.

Los estudios han demostrado que los resultados intermedios en la construcción del conocimiento son posibles a través del aprendizaje activo, los jóvenes avanzan por un camino de construcción de conocimiento, de almacenamiento del conocimiento y de absorción del conocimiento. Este proceso de construcción de conocimiento depende del conocimiento previo que tenga el aprendiz en donde el aprendiz pueda comprometerse con propio proceso de cambio en las bases cognitivas y pueda regularlo por sí mismo.

El aprendizaje colaborativo consiste en una situación de aprendizaje en la cual dos o más personas intentan aprender algo, juntos. A diferencia del aprendizaje individual, las personas involucradas en el aprendizaje colaborativo aprovechan los recursos, habilidades y talentos de otros (como preguntar sobre determinada información, evaluar ideas de otro, monitorear el trabajo de los demás, etc.). De una manera más clara, el aprendizaje colaborativo se basa en el modelo de conocimiento que se puede crear cuando existen miembros activos que pueden interactuar a partir de compartir experiencias y asumir roles asimétricos [13].

De la misma manera, el aprendizaje colaborativo se refiere a las metodologías y entornos en donde los aprendices se comprometen con una tarea común en donde cada uno depende de los demás. Esto incluye tanto un trabajo de conversaciones cara a cara aunque en la actualidad la interacción directa mediada por servicios asíncronos que proveen las nuevas tecnologías ha cambiado el concepto de la interacción directa y lo han actualizado [14]. De esta forma, se consideran en la actualidad como parte de este tipo de aprendizaje, los foros en línea, las salas de chats, los enlaces directos y otros servicios similares. Entre los métodos que permiten examinar los resultados que provienen de la adopción de estrategias de aprendizaje colaborativo incluyen análisis de conversaciones y análisis estadísticos de discursos.

Por estas razones, el aprendizaje colaborativo es mucho más útil cuando se usa con grupos de estudiantes que trabajan juntos buscando aprender, encontrar significado, resolver problemas o crear un artefacto o un producto de su propio aprendizaje [15]. 
De esta forma, el aprendizaje colaborativo pareciera redefinir el rol de la relación entre docente y estudiante $\mathrm{y}$, de manera particular, el papel del docente en un proceso de aprendizaje en el aula de clases cuyos resultados siempre parecieran ser mucho más benéficos que perjudiciales dada la interacción que se sucede durante el desarrollo de las actividades entre los mismos estudiantes. Entre las actividades de aprendizaje colaborativo se pueden incluir escritos colaborativo, proyectos en grupo, soluciones conjuntas de diferentes problemas, debates, equipos de estudio y muchas otras.

El aprendizaje colaborativo resulta ser una muy interesante forma de promover el pensamiento crítico entre los estudiantes. Las investigaciones indican que los estudiantes de manera individual pueden lograr niveles bastante altos de aprendizaje y de retención de información cuando trabajan en grupos [16]. Esto también permite el avance en el conocimiento para los instructores, facilitadores o acompañantes.

El aprendizaje cooperativo es un enfoque educativo que permite organizar actividades en el salón de clases de manera que se conviertan en experiencias académicas y sociales. Hay mucho más allá, en el aprendizaje cooperativo, que simplemente organizar a los estudiantes en grupos y se puede describir como una interdependencia positiva [17]. Los estudiantes deben trabajar en grupos para completar colectivamente las tareas con el fin de alcanzar objetivos académicos. A diferencia del aprendizaje que se da cuando el estudiante trabaja individualmente, el cual puede ser competitivo por naturaleza, los estudiantes que trabajan cooperativamente pueden aprovechar los recursos y habilidades de los demás.

El rol del docente cambia de dar información a facilitar el aprendizaje de los estudiantes. En un grupo, todos triunfan si el grupo triunfa. El aprendizaje cooperativo incluye metas como la exigencia intelectual, la creatividad, la mente abierta e incluye tareas de pensamiento de alto nivel. Cuando se incorpora el aprendizaje cooperativo como estrategia en el aula, es posible promover, entre los alumnos, interdependencia positiva, una fuerte relación entre los integrantes de los grupos, interacción mediada o directa pero frente a frente y totalmente síncrona, detección de las habilidades necesarias requerida en grupos pequeños y procesamiento en grupo [18]. Los estudiantes involucrados en procesos de aprendizaje cooperativo, tienden a trabajar mejor en grupo, a razonar mejor, a entender otros puntos de vista, a mejorar su autoestima, a debatir sanamente y a aprender a complementarse con los demás.

El modelo 4Q de preferencias de pensamiento es un modelo formulado por William Herrmann, quien siempre se preocupó por la forma como el cerebro explica las cosas de manera diferente en cada persona y por qué algunas cosas son más simples para unas personas que para otras. Acudiendo a la investigación sobre el cerebro desarrollada por diferentes científicos, Herrmann descubrió que existían cuatro patrones que explican la forma como el cerebro percibe y procesa la información. El modelo 4Q emerge como una metáfora válida, aceptada internacionalmente, para explicar estos modos cuatro modos de preferencias.

El modelo 4Q (Whole Brain ${ }^{\circledR}$ Thinking) es una metodología diseñada para ayudar a los pensadores, a los equipos y a las organizaciones a lograr los mejores beneficios de las personas que dirijan o de los integrantes que pertenezcan.

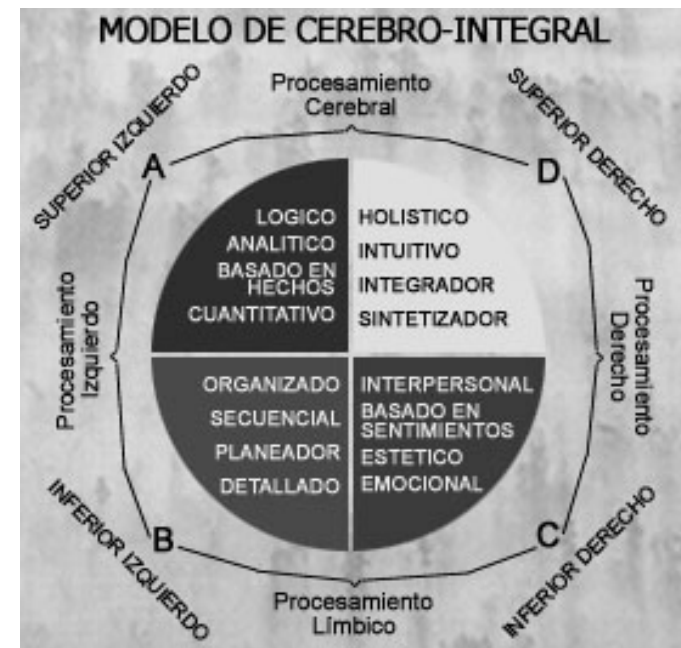

Figura 1. Modelo 4Q de preferencias de pensamiento - William Herrmann Fuente: http://herrmannlatin.com/hbdi.php (consulta: mayo 18 2017)

El modelo reconoce que mientras diferentes tareas requieren diferentes procesos mentales, y diferentes personas tienen diferentes formas de pensar, las organizaciones obtendrán mejores resultados cuando conozcan todo el espectro posible de pensamientos y enfoques que tienen los seres humanos [19].

Todas las personas tienen preferencias de pensamiento, algunas son muy fuertes, otras son más moderadas. Estas preferencias desarrollan ciertos niveles de dominación y no siempre se tiene consciencia de estas preferencias al punto que incluso dos personas pueden tener puntos de vista aparentemente distintos por no saber cuáles son sus preferencias de pensamiento. El modelo $4 \mathrm{Q}$ nos recuerda que cada uno tiene acceso a las cuatro formas de pensamiento pero una de ellas domina a los demás cuadrantes cuando sea necesario, cuando requiera adaptarse o cuando necesite tomar ventaja en determinada situación con el ánimo de mejorar el rendimiento y obtener mejores resultados [20]. La figura 1 presenta un esquema gráfico del modelo $4 \mathrm{Q}$ referenciado.

Según este modelo el cuadrante A corresponde al pensamiento lógico analítico lo cual permite que las personas con esta preferencia de pensamiento sean apropiadas para recolectar datos, analizarlos, entender cómo trabajan las cosas, juzgar ideas basadas en hechos y también para el razonamiento lógico. En el cuadrante B se ubica el pensamiento secuencial y por lo tanto aquellos que lo tienen como cuadrante preferente son muy hábiles para seguir instrucciones, para realizar trabajos plenamente orientados, para resolver un problema paso a paso así como para organizar e implementar una determinada solución.

El cuadrante C corresponde al pensamiento interpersonal, es el cuadrante social según el cual las personas tienen mayor capacidad para escuchar y expresar ideas, para buscar y encontrar aquello que es importante para las personas, para analizar las características de grupos de interacción y, en muchos casos, incluso para liderarlos. El cuatro cuadrante es el cuadrante D o imaginativo. En este cuadrante se ubican a las personas que son capaces de observar el paisaje y no se detienen a mirar solamente un árbol, toman la iniciativa y asumen retos por ellos mismos, son mucho más visuales y tienen un 
pensamiento que tiene un alto componente metafórico, poseen la capacidad de resolver creativamente los problemas y tienen un pensamiento que se distingue a largo plazo por sus características [21].

La enseñanza de la programación se ha ido constituyendo en el eje central de diferentes programas de formación no solamente por la necesidad de conocer y capitalizar las capacidades de los dispositivos modernos y aprovechar sus características, desde adentro, sino porque al incorporar a la solución de problemas una lógica que no es la nuestra, se fortalece nuestra lógica humana, dispersa, difusa y deliberativa y, por momento, incluso, imprecisa [22]. La enseñanza y el aprendizaje de la programación de computadores es la base de programas de formación profesional como la Ingeniería de Sistemas, según su enfoque curricular, en la cual se pretende concebir soluciones que permitan resolver problemas de la sociedad a partir de las capacidades que ofrece la tecnología moderna y desde un enfoque sistémico que permita ver el contexto completo de una determinada situación y no solamente una determinada situación problémica [23].

El aprendizaje de la programación pasa por tres instancias: el aprendizaje de la matemática que subyace a la programación, el aprendizaje de la lógica que posibilita la programación y el aprendizaje de la tecnología que cristaliza la programación [24]. Sin temor a equívocos, el modelo matemático que subyace a la programación se ha ido convirtiendo en un tema cada vez menos puesto en escena toda vez que la tecnología, con sus diferentes formas, cautiva a un nivel tal que termina seduciendo más la aplicación que el estudio y apropiación de los fundamentos desconociendo que la tecnología es transitoria mientras que las bases matemáticas son mucho más duraderas.

La lógica de programación se fundamenta en la apropiación de conceptos que, sin ser naturales en el ser humano, son los que permiten que se pueda hacer que un dispositivo logre, con gran eficiencia, lo que nosotros podríamos lograr pero si contamos con tiempo suficiente y si se nos acepta el permanente riesgo de cometer errores [25]. La tecnología asociada a la programación pasa por el filtro del conocimiento y apropiación de los lenguajes de programación, que es la instancia que más seduce particularmente a los jóvenes de hoy, de manera que termina buscándose en los cursos de programación un aprendizaje que puede ser efímero y cuyas bases se resienten cuando la tecnología cambia.

\section{Metodología}

Tal como ya se expuso en la introducción del presente artículo, esta investigación se desarrolló con los estudiantes del curso Programación II de Ingeniería de Sistemas y Computación de la Facultad de Ingenierías de la Universidad Tecnológica de Pereira durante los semestres I 2014, II 2014, I 2015, II 2015, I 2016 y II 2016. Para poder obtener resultados que se pudieran comparar y permitieran realizar inferencias confiables, desde el punto de vista investigativo, cada grupo de estudio se dividió en dos subgrupos: un subgrupo conformado por estudiantes que se categorizaron claramente a la luz de la categorización que provee el modelo $4 \mathrm{Q}$ de preferencias de pensamiento y en los cuales, a partir del diálogo y la interacción con ellos, se han podido identificar claramente las características de cada cuadrante dominante en los estudiantes. El otro subgrupo correspondió a estudiantes cuyo desempeño académico se analizaría de manera independiente de forma que se pudiera imitar, in situ, el método tradicional de enseñanza de la programación.

Durante cada semestre se hicieron tres evaluaciones escritas y cada una se asoció con un taller también escrito, de forma que cada nota parcial se lograra por la ponderación entre la evaluación escrita y el taller realizado. El peso porcentual de cada evaluación parcial fue del 20\%. La evaluación final tuvo un peso porcentual de $30 \%$ y el $10 \%$ restante (para completar el $100 \%$ de la nota definitiva) consistió en una nota 5.0 que se asignó a cada estudiante como elemento motivacional que le fue anunciado desde la primera clase y cuyo efecto fue inmediato.

En la $1^{\mathrm{a}}$ evaluación escrita y $1^{\mathrm{o}}$ taller se organizaron grupos de a 4 estudiantes; en la $2^{\text {a }}$ evaluación escrita y $2^{\circ}$ taller se organizaron grupos de 3 estudiantes teniendo en cuenta que algunos tuvieron que trabajar, por razones de la matemática de distribución, en grupos de 4 estudiantes como en las primeras pruebas. Es de anotar que se procuró al máximo que cada grupo, en las segundas pruebas, tuviera 3 estudiantes. En la $3^{\text {a }}$ evaluación escrita y $3^{\circ}$ taller se organizaron grupos de a 2 estudiantes (es decir, parejas) para lo cual se le permitió a los estudiantes que escogieran libremente un compañero de aquellos con los que había trabajado en el grupo de 4 personas.

La evaluación final se realizó de manera individual. Por su parte, los estudiantes independientes presentaron todas las pruebas escritas y los talleres de manera individual. La Tabla 1 presenta la distribución de los estudiantes en los diferentes grupos.

La Tabla 2 presenta la distribución detallada de los estudiantes de acuerdo a lo planteado en el diseño de la investigación y en consonancia con la reorganización de cada prueba.

Tabla 1.

Distribución de los estudiantes

\begin{tabular}{cccccc}
\hline \multirow{2}{*}{ Año } & Sem & $\begin{array}{c}\text { Tot } \\
\text { Ests }\end{array}$ & $\begin{array}{c}\text { Est } \\
\text { Indep }\end{array}$ & $\begin{array}{c}\text { Est } \\
\text { 4Q }\end{array}$ & $\begin{array}{c}\text { Grp } \\
\text { 4Q }\end{array}$ \\
\hline \multirow{2}{*}{2014} & I & 19 & 7 & 12 & 3 \\
& II & 22 & 6 & 16 & 4 \\
2015 & I & 23 & 7 & 16 & 4 \\
& II & 21 & 9 & 12 & 3 \\
2016 & I & 20 & 8 & 12 & 3 \\
& II & 21 & 9 & 12 & 3 \\
\hline
\end{tabular}

Fuente: Elaboración propia

Est 4Q: Estudiantes que formaron parte de los grupos 4Q Grp 4Q: Cantidad de grupos 4Q

Tabla 2.

Distribución detallada de estudiantes

\begin{tabular}{cccccc}
\hline Año & Sem & $\begin{array}{c}\mathbf{1}^{\mathbf{0}} \mathbf{P} \\
\text { (4 est) }\end{array}$ & $\begin{array}{c}\text { Grupos } \\
\mathbf{2}^{\mathbf{0}} \mathbf{P} \\
\text { (3 est) }\end{array}$ & $\begin{array}{c}\mathbf{3}^{\mathbf{0}} \mathbf{P} \\
(\mathbf{2} \text { est) }\end{array}$ & $\begin{array}{c}\text { EF } \\
\text { (1 est) }\end{array}$ \\
\hline \multirow{2}{*}{2014} & I & 3 & 4 & 6 & 12 \\
& II & 4 & $4+1(4)$ & 8 & 16 \\
2015 & I & 4 & $4+1(4)$ & 8 & 16 \\
& II & 3 & 4 & 6 & 12 \\
2016 & & & & & \\
& I & 3 & 4 & 6 & 12 \\
\hline
\end{tabular}

Fuente: Elaboración propia 
Tal como se explicó, en este mismo numeral, la nota definitiva en cada curso se obtuvo de la siguiente forma: la nota que se obtenía de la prueba escrita y del taller tenía cada una un peso de $10 \%$. La nota del examen final tenía un peso porcentual del $30 \%$ y un $10 \%$ que equivalía a una nota de 5.0 con carácter motivacional. De esta forma, la nota final se obtuvo así:

$$
\begin{aligned}
& \begin{array}{llll}
1^{\mathrm{o}} \text { parcial }(10 \%) & + & 1^{\circ} \text { taller }(10 \%) & =20 \% \\
1^{\mathrm{o}} \text { parcial }(10 \%) & + & 1^{\circ} \text { taller }(10 \%) & =20 \%
\end{array} \\
& \begin{array}{llll}
1^{\mathrm{o}} \text { parcial }(10 \%) & + & 1^{\circ} \text { taller }(10 \%) & =20 \% \\
1^{\mathrm{o}} \text { parcial }(10 \%) & + & 1^{\circ} \text { taller }(10 \%) & =20 \%
\end{array} \\
& \text { Evaluación Final } \quad=30 \% \\
& \text { Nota Motivacional (5.0) } \\
& \text { Total } \quad \rightarrow \quad 100 \%
\end{aligned}
$$

Las evaluaciones escritas se trabajaron desde la perspectiva del aprendizaje cooperativo y los talleres se trabajaron desde la perspectiva del aprendizaje colaborativo. Siempre se hicieron primero los talleres y, al final del período, se realizaron los parciales. A todos los estudiantes, los que pertenecieron a los grupos $4 \mathrm{Q}$ y los independientes, se les asesoró y se les acompañó con la misma voluntad y por todos los medios posibles. La exposición magistral y las explicaciones fueron las mismas para todos.

\section{Resultados}

La Tabla 3 presenta el promedio de las evaluaciones de las pruebas escritas y los talleres así como de la evaluación final obtenida tanto por los estudiantes que participaron en los grupos $4 \mathrm{Q}$ como por los estudiantes que trabajaron de manera independiente. Debe tenerse en cuenta que las notas obtenidas tanto por las pruebas escritas como por los talleres de los grupos $4 \mathrm{Q}$ se asignaron a cada grupo completo por lo tanto el cálculo de su promedio se hace sobre la cantidad de grupos que participaron dentro de cada evaluación escrita y cada taller. Debe recordarse que la nota parcial se obtiene obteniendo la semisuma de la evaluación escrita y el taller.

Debe recordarse que la nota definitiva que aparece es el $90 \%$ pues el otro $10 \%$ corresponde al valor 5.0 que se reconoció a todos los estudiantes como factor motivacional. Al finalizar cada curso se solicitó una opinión anónima de cada estudiante y solo se le pidió que escribiera si había participado de la investigación como estudiante independiente o como estudiante integrante de un grupo 4Q. Los resultados se clasificaron como:

- Altamente Favorables (AF) aquellos estudiantes que manifestaron gran complacencia por la participación en el proceso y que consideran que aprendieron a programar

- Favorables (F) aquellos estudiantes que manifestaron complacencia en la participación en el proceso y consideran que no aprendieron a programar tanto como esperaban

- Poco Favorables (PF) aquellos estudiantes que consideraron que su curso de programación estuvo dentro de los cauces normales y que su aprendizaje estuvo dentro de lo normal

- Desfavorables (DF) aquellos estudiantes que consideran que no les gustó la metodología en la cual le correspondió participar y que creen que hubieran aprendido mucho más si hubieran estado en el otro grupo

- Altamente Desfavorables (ADF) aquellos estudiantes que

\begin{tabular}{|c|c|c|c|c|c|c|c|c|}
\hline Año & $S$ & Grp & $1^{\circ} \mathrm{P}$ & $2^{\circ} \mathrm{P}$ & $3^{\circ} \mathbf{P}$ & EF & ND* & Dif. \\
\hline \multirow{4}{*}{2014} & \multirow[b]{2}{*}{ I } & Indep & 2.5 & 2.7 & 3.1 & 3.4 & 2.68 & \multirow[b]{2}{*}{1.20} \\
\hline & & 40 & 4.5 & 4.3 & 4.3 & 4.2 & 3.88 & \\
\hline & \multirow{2}{*}{ II } & Indep & 3.1 & 3.0 & 3.4 & 3.2 & 2.86 & \multirow{2}{*}{0.94} \\
\hline & & $4 Q$ & 4.6 & 4.3 & 4.1 & 4.0 & 3.80 & \\
\hline \multirow{4}{*}{2015} & & Indep & 3.4 & 3.5 & 3.7 & 3.9 & 3.29 & \multirow{2}{*}{0.69} \\
\hline & 1 & $4 Q$ & 4.7 & 4.5 & 4.4 & 4.2 & 3.98 & \\
\hline & \multirow{2}{*}{ II } & Indep & 3.5 & 3.5 & 3.6 & 3.6 & 3.20 & \multirow[b]{2}{*}{0.66} \\
\hline & & $4 Q$ & 4.6 & 4.5 & 4.2 & 4.0 & 3.86 & \\
\hline \multirow{4}{*}{2016} & \multirow{2}{*}{ I } & Indep & 3.6 & 3.7 & 4.0 & 4.1 & 3.49 & \multirow{2}{*}{0.52} \\
\hline & & $4 Q$ & 4.7 & 4.6 & 4.3 & 4.3 & 4.01 & \\
\hline & \multirow{2}{*}{ II } & Indep & 3.3 & 3.5 & 3.8 & 3.9 & 3.29 & \multirow{2}{*}{0.53} \\
\hline & & $4 Q^{1}$ & 4.5 & 4.4 & 4.2 & 4.0 & 3.82 & \\
\hline \multirow{2}{*}{\multicolumn{2}{|c|}{ Promedios }} & Indep & 3.23 & 3.31 & 3.60 & 3.68 & 3.135 & \multirow{2}{*}{0.75} \\
\hline & & $4 Q$ & 4.60 & 4.43 & 4.25 & 4.11 & 3.891 & \\
\hline
\end{tabular}
no les gustó el grupo en el cual les tocó y que creen que no aprendieron nada de programación.
Tabla 3.

Promedio de notas obtenidas

\begin{tabular}{|c|c|c|c|c|c|c|c|c|c|}
\hline Año & $\mathrm{S}$ & Grp & $\mathbf{A F}$ & $\mathbf{F}$ & PF & DF & ADF & Ests & Tot \\
\hline \multirow{4}{*}{2014} & \multirow{2}{*}{ I } & Indep & 0 & 2 & 2 & 3 & 0 & 7 & \multirow{2}{*}{19} \\
\hline & & $4 Q$ & 8 & 3 & 1 & 0 & 0 & 12 & \\
\hline & \multirow{2}{*}{ II } & Indep & 0 & 2 & 2 & 2 & 0 & 6 & \multirow{2}{*}{22} \\
\hline & & $4 \mathrm{Q}$ & 11 & 4 & 1 & 0 & 0 & 16 & \\
\hline \multirow{4}{*}{2015} & \multirow{2}{*}{ I } & Indep & 0 & 0 & 3 & 3 & 1 & 7 & \multirow{2}{*}{23} \\
\hline & & $4 Q$ & 7 & 5 & 3 & 1 & 0 & 16 & \\
\hline & \multirow{2}{*}{ II } & Indep & 0 & 1 & 2 & 5 & 1 & 9 & \multirow{2}{*}{21} \\
\hline & & $4 Q$ & 7 & 3 & 2 & 0 & 0 & 12 & \\
\hline \multirow{4}{*}{2016} & \multirow{2}{*}{ I } & Indep & 1 & 1 & 5 & 1 & 0 & 8 & \multirow{2}{*}{20} \\
\hline & & $4 \mathrm{Q}$ & 8 & 2 & 1 & 1 & 0 & 12 & \\
\hline & \multirow{2}{*}{ II } & Indep & 0 & 0 & 3 & 3 & 3 & 9 & \multirow{2}{*}{21} \\
\hline & & $4 Q$ & 9 & 1 & 1 & 1 & 0 & 12 & \\
\hline \multirow{2}{*}{\multicolumn{2}{|c|}{ Totales }} & Indep & 1 & 6 & 17 & 17 & 5 & 46 & \multirow{2}{*}{126} \\
\hline & & $4 \mathrm{Q}$ & 50 & 18 & 9 & 3 & 0 & 80 & \\
\hline
\end{tabular}

Fuente: Elaboración propia

ND* = Nota Definitiva (90\%)

Tabla 4.

Opinión de los estudiantes

La Tabla 4 presenta un resumen de los resultados obtenidos luego de tabular las respuestas de los estudiantes.

Los estudiantes que participaron en los grupos $4 \mathrm{Q}$ plantearon como grandes ventajas la posibilidad de aprender de otros compañeros, la opción que tienen de asumir responsabilidades dentro de un grupo al momento de hacer los parciales, la interacción con los compañeros en el desarrollo de los talleres y la cohesión que se genera entre los mismos estudiantes incluso cuando el grupo, que comienza con 4 integrantes, va disminuyendo. Por su parte los estudiantes que participaron en la investigación de manera independiente denotaron más las desventajas entre las que se cuenta la orfandad que perciben al no poder recurrir a otra persona que no sea el profesor aunque asumen que, trabajando en solitario y por su propia cuenta, consideran que es posible que puedan aprender más, según sus propias palabras. Precisamente esta investigación busca determinar si eso es posible o no.

\section{Discusión}

La adopción del modelo 4Q en el desarrollo de este proyecto de investigación obedece a la necesidad de tener unos 
elementos de juicio con rigor científico que permitan categorizar a los estudiantes para organizarlos en el formato de grupos de trabajo. Normalmente la utilización de esta estrategia en el aula es aleatoria pues el docente deja que los estudiantes se organicen con los compañeros que tengan al lado. Si bien, esta es la forma como normalmente se ha hecho, debe admitirse que la organización del aula en grupos de trabajo requiere una participación más activa del docente.

Existen diferentes modelos a los cuales se puede acudir al momento de organizar grupos de trabajo dentro del aula. El modelo 4Q de preferencias de pensamiento pareciera permitir una identificación de los estudiantes de manera que se puedan complementar sus habilidades y sus capacidades en pro del logro de unos determinados objetivos de aprendizaje. En este estudio, por razones propias de la carrera Ingeniería de Sistemas, se consideró que los cuatro cuadrantes dominantes generan perfiles que son de alta relación no sólo con el perfil profesional y laboral de los Ingenieros de Sistemas sino con las competencias que deben desarrollarse para su desempeño. De allí que unir a un Lógico con un Secuencial, un Social y un Imaginativo, hablando en términos del modelo en mención, lleva a pensar que pueden complementarse y lograr objetivos comunes para beneficio del grupo y para beneficio de cada uno.

Se ha acudido también a los principios establecidos en los enfoques de aprendizaje cooperativo y aprendizaje colaborativo desde dos perspectivas: la realización de los talleres y el desarrollo y resolución de las pruebas escritas. En la realización de los talleres se ha pensado en el aprendizaje colaborativo dado que, en éste, se establecen unas reglas mínimas de trabajo que comienzan por la designación de un líder dentro del grupo cuya responsabilidad es conducir el proceso de resolución de los problemas que se planteen.

Dado que un taller pretende que los estudiantes, mutuamente, se colaboren para que aprendan a resolver problemas desde la óptica de la lógica computacional, complementado con el debido acompañamiento del docente, se ha considerado que esta es la estrategia adoptar. Debe acotarse que, a pesar de que se cuente con un líder dentro del grupo en la conducción de la resolución de talleres, todos los estudiantes tienen la posibilidad de preguntar, participar, cuestionar y proponer soluciones dentro del marco de la construcción de las respuestas del taller.

Por su parte, el aprendizaje cooperativo ha sido la estrategia que se ha adoptado en la realización de las pruebas escritas dado que, como estas tienen un tiempo un poco más limitado y más preciso, la participación activa de todos es muy importante y eso invita a pensar que la labor del líder, tan útil en lo colaborativo, podría no ser tan efectiva en lo cooperativo. De allí que, siempre se acudió primero a resolver talleres $\mathrm{y}$, posteriormente finalizando lo que podríamos llamar como período, se realizaron las pruebas escritas con asignación específica de funciones de cada uno de los participantes. Es de anotar que la asignación de funciones en las pruebas escritas implica la aceptación de responsabilidades en el desarrollo de la construcción de respuestas de ellas.

La diferencia fundamental entre lo colaborativo y lo cooperativo es que en la primera se pueden delegar funciones a partir de la opinión de un líder quien es el que conduce el proceso; la segunda se basa en la participación activa de todos los integrantes del grupo de manera que cada uno se haga responsable de determinadas partes en la actividad que, para este caso, son las pruebas escritas. Se notó en los estudiantes gran aceptación del modelo 4Q cuando se les explicó y cuando se les ubicó dentro de algunos de los cuadrantes dominantes. De la misma manera se notó complacencia de la conformación de los grupos y, a partir de la observación directa, se percibió un ambiente de alta camaradería y colaboración tanto en los talleres como en las pruebas escritas.

La reducción de los grupos, en la medida en que avanzaban las semanas y se adoptaban nuevos temas, nuevos talleres y nuevas pruebas escritas, se pensó como una forma de ir logrando que los estudiantes se fueran desprendiendo, si el término es posible, de sus respectivos líderes en cada grupo 4Q y fueran asumiendo responsabilidades propias a partir de lo visto en las primeras semanas. Los grupos conformados por los cuatro perfiles preferentes se notaron bastante activos y muy sincronizados. Los grupos conformados por solo tres perfiles no tuvieron la armonía de colaboración y cooperación que se esperaba.

Las parejas de trabajo trabajaron muy bien y cada par de estudiantes se sintonizaron de una forma bastante llamativa. Es de anotar que fue esta la única oportunidad en la cual se le permitió al estudiante escoger a su pareja tomando como referencia a los compañeros con los cuales interactuó en el grupo inicial. La presentación de la evaluación final de manera individual fue, para los estudiantes que venía de grupos $4 \mathrm{Q}$, una oportunidad de probarse a sí mismos qué tanto habían aprendido. Los resultados cuantitativos se evaluarán más adelante.

Se quiso mantener un peso porcentual equitativo entre las evaluaciones parciales y se le quiso conceder un peso mayor a la evaluación final dado que en esta prueba no sólo se evalúan todos los temas vistos durante el semestre sino que se realiza de manera individual tanto para los estudiantes que integraron los grupos 4Q como los estudiantes que, desde el principio, trabajaron individualmente. El elemento motivacional se concedió a través de un $10 \%$ de la nota definitiva que, para todos los estudiantes, correspondió a una nota de 5.0. Para lograr dicho efecto motivacional, se anunció esto a los alumnos desde el primer día de clase.

El promedio de las notas obtenidas en cada evaluación y su progresión en el tiempo mantienen algunos elementos constantes que referiremos a continuación. De una parte las notas de los estudiantes independientes, sin excepción, en la medida en que avanzaban en el tiempo se iban incrementando posiblemente debido al gran esfuerzo individual que hacía cada estudiante independiente y que tal vez tiene que ver con la solidez de la fundamentación en las primeras semanas pues en éstas también el estudiante estaba solo. En el promedio de las notas de los grupos 4Q y de sus derivados, se nota que las primeras notas son significativamente altas aunque con el paso del tiempo se van disminuyendo en la medida en que se avanza entre las evaluaciones parciales. Esta constante también se mantuvo durante todos los semestre en los cuales se realizó la investigación tal como se muestra en la Tabla 3.

La diferencia de las notas siempre fue positiva a favor de los estudiantes de los grupos 4Q. De la misma manera la nota más baja de los grupos $4 \mathrm{Q}$ siempre fue más alta que la nota más alta 
de los estudiantes independientes. También se observa en la nota definitiva, de la cual se excluyó el 10\% motivacional por ser la misma nota para todos, que siempre el promedio de los estudiantes $4 \mathrm{Q}$ es más alto que el promedio de los estudiantes independientes. Eso pareciera indicar que, al enfrentarse a la misma evaluación, los estudiantes que participaron en los grupos $4 \mathrm{Q}$ parecieran tener más claros tanto los conceptos como su aplicación, que los que trabajaron durante todo el semestre de manera independiente. Es de anotar que los talleres, las pruebas escritas y la evaluación final siempre fue la misma para todos los estudiantes cualquiera que fuere el subgrupo con el cual hubieren trabajado.

En un análisis vertical, se nota también que el promedio comparativo entre las diferentes evaluaciones parciales es mucho más alto en los estudiantes que participaron en los grupos $4 \mathrm{Q}$ que en los estudiantes que trabajaron de manera independiente. Debe anotarse que, a pesar de que las notas no siempre reflejan el resultado del conocimiento, no se puede desconocer que estando inmersos en las mismas clases magistrales, los mismos talleres y las mismas pruebas escritas, las diferencias en las notas sugieren conclusiones que saltan a la vista y que, a partir de la observación del docente, dan fe de que los estudiantes que participaron en grupos $4 \mathrm{Q}$ fueron aprendieron mejor los conceptos teóricos, y su aplicación, de la lógica de programación.

La opinión de los estudiantes que participaron en los grupos $4 \mathrm{Q}$ y el balance que éstos hacen del proceso investigativo es Altamente Favorable (AF) tal como se muestra en la Tabla 4. Los estudiantes independientes tienden, en sus opiniones, a ser Poco Favorables (PF) y Desfavorables (DF) en contraposición con la opinión de los estudiantes que pertenecieron a los grupos $4 \mathrm{Q}$. Es de anotar que la interacción, la posibilidad de despejar dudas con pares de su mismo nivel, la opción de participar activamente y enunciar sus opiniones libremente son algunas de las razones que han esgrimido los estudiantes $4 \mathrm{Q}$ para destacar las ventajas de la estrategia adoptada para el desarrollo de esta investigación.

\section{Conclusiones}

La adopción de enfoques como el aprendizaje colaborativo y el aprendizaje cooperativo permiten que se fortalezca la relación e interacción entre estudiantes y, al tiempo, posibilita la relación pedagógica entre el docente y sus alumnos a la luz de las dudas que se presentan bien sean en conjunto o bien de manera individual. El hecho de que un estudiante pueda contar con un par académico de su mismo nivel pareciera permitir un camino bastante llano para exponer sus dudas incluso en el caso en que se mejore notoriamente la interacción alumno profesor.

El modelo 4Q es una forma muy interesante de conocer un poco más a los estudiantes en su visión del mundo y en la forma como lo leen y como lo interpretan. Es posible que algunos cuadrantes preferentes tengan mayor sintonía que otros pero todo pareciera indicar que conformar grupos de trabajo basados en este modelo es una estrategia que enriquece a cada uno y, además, fortalece el concepto de "grupo de trabajo" a la luz de una temática específica y de unos objetivos de aprendizaje definidos.
La Ingeniería de Sistemas se nutre de los cuatro perfiles del modelo $4 \mathrm{Q}$ dado que en algún momento el egresado ha de tener una alta componente lógica (para buscar soluciones), una alta componente secuencial (para implementarlas), una alta componente social (para compartirlas) y una alta componente imaginativa (para encontrar nuevos caminos de aplicación). Debe anotarse que cada uno de los perfiles del modelo 4Q se complementan y han de aflorar en momentos determinados. Esto permite pensar en que podría ser este modelo una buena base para establecer las líneas de formación y profundización en un programa como Ingeniería de Sistemas y, a partir de un análisis científico, poder orientar a los estudiantes por el camino que mejor coincida con su cuadrante preferente, aunque este es tema de otro artículo.

La realización de pruebas escritas y talleres en grupos que cada vez se reducen hasta llegar a la individualidad es una estrategia que, a decir de los mismos estudiantes, ha gustado mucho pues poco a poco y en la medida en que los grupos van disminuyendo en su cantidad de integrantes, cada estudiante se va encontrando en situaciones evaluativas en las cuales son sus propias responsabilidades en unión con sus capacidades las que permiten que se enfrente solo y demuestre lo que ha aprendido. Por esta razón siempre es importante que en estos procesos no falte ni la observación detallada de cada alumno por parte del docente ni la opinión, preferiblemente escrita, por parte de los estudiantes al final del proceso para retroalimentar y tener un camino cualitativo, además de las valoraciones cuantitativas, que permitan ajustar estas investigaciones para poder socializar sus resultados.

La investigación en el aula en programas de ingeniería permite explorar nuevas formas de aprovechar toda la teoría que se deriva de las Ciencias de la Educación y ponerla al servicio de las necesidades de conocimiento de ingenieros en formación. Para ello los ingenieros docentes deben formarse tanto en su conocimiento disciplinar como en estas áreas que son las que proveen los elementos de juicio necesarios, las teorías, estrategias, enfoques y metodologías que permitirán que se alcancen los logros de aprendizaje de sus alumnos por caminos más efectivos y menos complejos.

\section{Referencias}

[1] Ausubel, D., Sicología educativa: Un punto de vista cognoscitivo. Ciudad de México: Trillas, 1986, 686 P.

[2] Gonzalez-García, F., Los mapas conceptuales de Novak como instrumentos para la investigación en didáctica de las ciencias experimentales. Enseñanza de las Ciencias, 10(2), pp. 148-158, 1992.

[3] Calvani, A., Connectivism: New paradigm or fascinating potpourri. Journal of e-Learning and Knowledge Society, 4(1), pp. 247-252, 2008. DOI: $10.20368 / 1971-8829 / 268$

[4] Bruner, J.S., Actos de significado: Mas allá de la revolución cognitiva. Madrid: Alianza Editorial. 2009, 130 P.

[5] Attard, A., Di Ioio, E. and Geven, K., Student centered learning. An insight into theory and practice. Bucarest: Lifelong learning programme - European Community, 2010.

[6] Ausubel, D., Psychology of meaningful verbal learning: An introduction to school learning. New York: Grune \& Straton. 1963, 780 P.

[7] Trejos-Buriticá, O., Significado y competencias. Pereira: Papiro, 2013, $130 \mathrm{P}$.

[8] Diaz-Barriga, F., Estrategias docentes para un aprendizaje significativo. México: McGraw Hill, 2005, 145 P.

[9] Bruner, J.S., Hacia un teoría de la instrucción. Ciudad de México: Hispanoamericana, 1969, $126 \mathrm{P}$. 
[10] Bruner, J.S., El proceso de la Educación. Ciudad de México: Editorial Hispanoamericana, 1963, $200 \mathrm{P}$

[11] Bonwell, C. and Eison, J., Active learning: Creating excitement in the classroom. ASHEERIC Higher Education Report No 1(1), 1991. DOI: $10.1177 / 1052562905283346$

[12] Prince, M., Does active learning work? Journal Engineering Education, 93(3), pp. 223-231, 2004.

[13] Dooly, M., Constructing knowledge together, en: Dooly, M., Telecollaborative language learning. A guidebook to moderating intercultural collaboration online, Bern: Peter Lang, 2008, pp. 21-45.

[14] Small, G., El cerebro digital. Madird: Editorial Urano. 2011.

[15] Barriga-Arceo, F. y Hernandez-Rojas, G., Estrategias docentes para un aprendizaje significativo: Una interpretación constructivista. Ciudad de México: McGraw Hill Interamericana, 2002, 241 P.

[16] Martín-Moreno-Cerrillo, Q., Aprendizaje colaborativo y redes de conocimiento. IX Jornadas Andaluzas de Organización y Dirección de Instituciones Educativa, Grananda, España, 2004, pp. 55-70.

[17] Adams, A., Cooperative learning effects on the classroom . Michigan: Northern Michigan University, November 26, 2013.

[18] Mabrouk, P., Cooperative learning and active learning: Models from the analytical sciences. ACS Symposium series 970, 2007, pp. 34-53.

[19] Lumsdaine, E. and Lumsdaine, M., Creative solving problem: Thinking skills for a changing world. New York: McGraw Hill, 2005.

[20] Herrmann, W., Creative brain. New York: The Ned Herrmann Group, 1988, $456 \mathrm{P}$.

[21] Herrmann, W., The whole brain. New York: McGraw Hill. 2000, 334 P.

[22] Trejos-Buriticá, O., La esencia de la lógica de programación. Pereira: Papiro, 2000, 366 P.

[23] Blanchard, B., Ingeniería de sistemas. Madrid (España): Isdefe, 2000, $256 \mathrm{P}$.

[24] Trejos-Buriticá, O.I., Fundamentos de programación. Pereira: Papiro. 2006, $130 \mathrm{P}$.

[25] Cooper, D., Locos por la tecnología. México: Editorial Limusa, 2004, $150 \mathrm{P}$
O.I. Trejos-Buriticá, es Ing. de Sistemas. Esp. en Instrumentación Física. MSc en Comunicación Educativa. PhD en Ciencias de la Educación. Docente de planta, de la Facultad de Ingenierías, Universidad Tecnológica de Pereira, Colombia. Ex director de Ingeniería de Sistemas y Computación. Ex decano Facultad de Ingenierías, Universidad Tecnológica de Pereira, Colombia.

ORCID: 0000-0002-3751-6014 